\title{
Cellular Function and Regulation of the Translationally Controlled Tumour Protein TCTP
}

\author{
Ulrich-Axel Bommer*
}

Graduate School of Medicine and Illawarra Health and Medical Research Institute, University of Wollongong, Wollongong, NSW 2522, Australia

\begin{abstract}
The 'translationally controlled tumour protein' TCTP was originally discovered 30 years ago by researchers interested in proteins regulated at the translational level. Cloning and sequencing confirmed the conservation of this protein among all eukaryotic kingdoms, but did not reveal any functional clue, and TCTP was listed in the databases as a 'family' of its own. The functional characterisation of this protein extended over more than a decade, leading to a plethora of individual functions and interactions that have been ascribed to this protein. A major addition to the functional characterisation of TCTP was the identification in 1995 of its histamine releasing factor (HRF) activity in allergic conditions, which for the first time described an extracellular activity for TCTP in human disease. This triggered a host of additional publications aimed at characterising this HRF activity, which are discussed in other articles of this issue. Another milestone in the elucidation of TCTP's function was the demonstration of its anti-apoptotic activity in 2001. Evidence is also accumulating for a role of TCTP in the cell cycle and in early development. This article provides an overview of the main cellular activities of TCTP. The second part will summarise our current knowledge on the mechanisms involved in regulating intracellular TCTP levels.
\end{abstract}

Keywords: TCTP, histamine releasing factor, fortilin, mitotic regulation, anti-apoptotic protein, mTORC1 signalling, tumour protein, translational regulation.

\section{INTRODUCTION}

\section{The Translationally Controlled Tumour Protein TCTP - History of Discovery}

The first reports on the translationally controlled tumour protein TCTP date back about 30 years. The protein was discovered independently by three groups studying proteins that are regulated at the translational level of gene expression. George Thomas investigated proteins that are regulated in response to mitogenic stimulation of mouse fibroblasts and identified a translationally controlled protein 'Q23' [1]. The group of George Brawerman (Boston) studied mRNAs that are abundantly represented in untranslated, nonribosomal mRNP particles, and among these identified the mRNA for a protein they called 'P21' [2]. In Heinz Bielka's group (Berlin) the protein 'P23' was found to be preferentially synthesized in exponentially growing vs. serumstarved Ehrlich ascites tumour cells [3]. The first cDNA sequences of the mouse [4] and human [5] protein where published around this time, and in the latter paper, the protein was termed 'translationally controlled tumour protein', since it was cloned from a human mammary tumour. The additional designations 'histamine releasing factor, HRF' [6] and 'fortilin' [7] were proposed later by the groups who discovered the histamine release and anti-apoptotic activities of

*Address correspondence to this author at the Graduate School of Medicine, University of Wollongong, Wollongong, NSW 2522, Australia; Tel: +61-2-4221-5138; Fax: +61-2-4221-4341;

E-mail: ubommer@uow.edu.au
TCTP, respectively. However, since these terms relate only to a specific activity of this multifunctional protein, the older, more unspecific name TCTP is still being used at large.

\section{Molecular Structure and Conservation of TCTP}

The cloning and sequencing did not provide any clue about the functional importance of TCTP, since no similarity to other proteins or functional domains was discovered, and thus the protein was listed as a 'family' on its own in the databases. As more and more TCTP sequences were deposited in the database, it became clear that TCTP is highly conserved among all eukaryotic kingdoms (reviewed in [8]). For a recent detailed sequence comparison and phylogenetic analysis see Hinojosa-Moya et al. [9].

Elucidation of the first 3D structure of the TCTP from the fission yeast $S$. pombe [10] demonstrated that the TCTP molecule consists of three distinct domains, the core $\beta$-sheet domain, an $\alpha$-helical domain and a flexible loop structure (Fig. 1). Many additional TCTP structures subsequently deposited in databases, confirmed that this principal structure is highly conserved in evolution. The first NMR structure analysis also revealed the similarity of the highly conserved $\beta$-sheet domain of TCTP with Mss4/Dss4 proteins, which bind to the GDP/GTP-free form of Rab proteins [10]. This discovery indicated that TCTP might be related to GTPaseassociated proteins and two examples of such functional activity will be discussed below. 


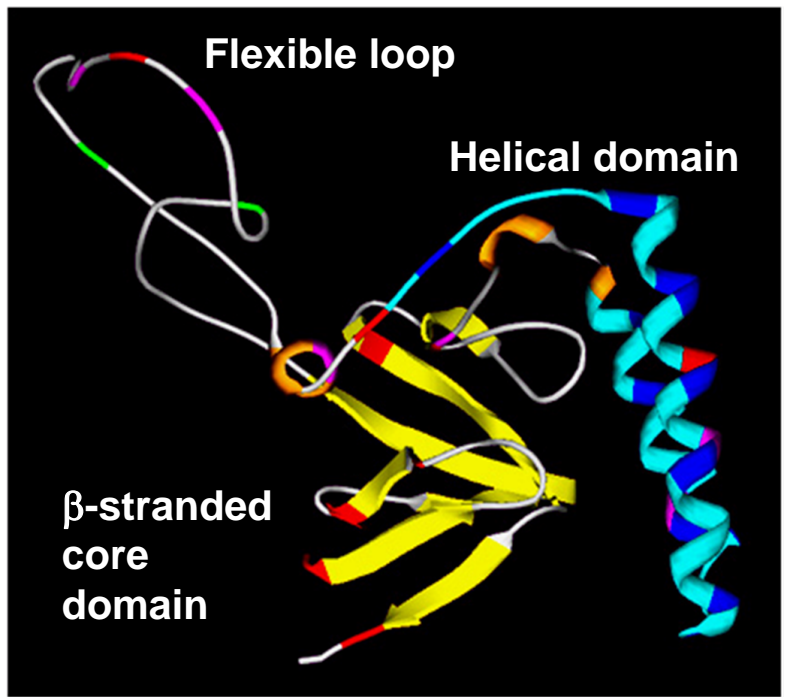

Fig. (1). Three-dimensional structure of the TCTP protein. The ribbon structure of fission yeast TCTP as originally published by Thaw et al. [10] is shown (PDB: 1H6Q). The domain structure is indicated. The $\beta$-stranded core domain is shown in yellow, and the $\alpha$-helical domain region is marked in blue. The most conserved residues are labelled in red or magenta. Green indicates the positions of the serine residues phosphorylated by the mitotic kinase Plk-1 [19].

This is the only indication of a functional activity, which arose from structural studies of TCTP. However, a plethora of additional functional activities has been described since by numerous research groups. It is the aim of this article to provide a current overview on the many molecular associations and cellular functions of TCTP, as well as on the mechanisms involved in the regulation of cellular TCTP levels.

\section{CELLULAR FUNCTIONS OF TCTP}

\section{Interaction of TCTP with the Cytoskeleton and its Role in Cell Division}

\section{- TCTP-A Non-Canonical $\mathrm{Ca}^{++}$-Binding Protein}

One of the earliest demonstrations on a functional activity for TCTP was the report on the $\mathrm{Ca}^{++}$-binding activity of trypanosome TCTP by Haghigat \& Ruben [11]. Since then, many groups have demonstrated $\mathrm{Ca}^{++}$-binding of TCTP by means of the $\mathrm{Ca}^{++}$-overlay assay (see e.g. [12]), even though the TCTP sequence does not harbor a canonical $\mathrm{Ca}^{++}$-binding motif.

In 2007, two detailed studies have been published aimed at characterising the $\mathrm{Ca}^{++}$-binding site in TCTP. Feng and co-workers [13] used multi-dimensional NMR spectroscopy to determine the solution structure of human TCTP and to identify the $\mathrm{Ca}^{++}$-binding site. They concluded that the weak binding site involves the residues N131, Q132 and D150, located in the $\beta$-stranded core domain close to the connection with the $\alpha$-helical domain. In contrast, Graidist et al. [12] employed a combination of several methods to study the $\mathrm{Ca}^{++}$-binding activity of TCTP. They performed a detailed mutational analysis, leading them to conclude that the residues E58 and E60, located in the floppy loop of the TCTP structure, are involved in $\mathrm{Ca}^{++}$-binding. These two appar- ently differing results might be reconciled, if there is more than one binding site for $\mathrm{Ca}^{++}$in the TCTP molecule. However, more studies will be necessary to resolve this question.

\section{- Interaction with the Cytoskeleton}

The first intracellular function described for TCTP, i.e. microtubule binding and stabilisation, was unveiled by our laboratory [14]. We demonstrated that TCTP (P23) associates with microtubules in a cell cycle-dependent manner. We identified the tubulin binding domain of TCTP, which is largely identical with the $\alpha$-helical domain of the 3D structure (Fig. 1). Overexpression of TCTP resulted in growth retardation of cells, microtubule stabilisation and alteration of cell morphology.

A recent, more detailed study into the interaction of TCTP with the cytoskeleton revealed that TCTP also interacts with actin filaments; it localises to a subset of actin-rich fibers in migrating cells [15]. This observation is corroborated by another report on a domain in TCTP, similar to the actin-binding domain in cofilin [16]. Bazile et al. [15] also demonstrated that TCTP's interaction with the microtubules is transient, and that it does not behave as a bona-fide microtubule-associated protein.

\section{- Binding to the Mitotic Spindle and Mitotic Phosphory- lation of TCTP}

In our study we reported that TCTP (P23) is bound to the mitotic spindle, but is detached from the spindle during metaphase-anaphase transition [14]. TCTP binding to the spindle, predominantly to the poles, has since been confirmed by reports from other laboratories [17,18]. It is anticipated that TCTP is involved in stabilising spindle microtubules [17,19], and the observed detachment of TCTP from the spindle might be important for the metaphase-anaphase transition. We also observed that TCTP is subject to mitotic phosphorylation (M. Lee, Y. Gachet and U.A. Bommer, unpublished results), and we anticipated that this phosphorylation event is necessary for the detachment of TCTP from the spindle microtubules. The protein kinase involved in TCTP phosphorylation was subsequently characterised by Yarm [19] as the mitotic polo-like kinase Plk-1. He also identified two phosphorylation sites for Plk-1 in the flexible loop of the TCTP structure (Fig. 1). Expression of a TCTP protein mutated in these sites led to severe disturbance of mitotic progression and to the formation of multinucleated cells. The phosphorylation of TCTP by Plk-1 has since been studied by another laboratory [20], and it has been proposed as a marker for the action of anti-cancer drugs, targeting Plk-1 [21].

\section{- TCTP as a General Mitotic Regulator}

There is an increasing body of evidence indicating that TCTP is indeed an important mitotic regulator, and mechanisms other than stabilisation of spindle microtubules have been proposed to play a role in this activity. A range of nuclear proteins involved in mitotic progression have been reported to interact with TCTP, and either to regulate TCTP levels or being regulated by TCTP.

Chfr is a checkpoint protein that plays an important role in cell cycle progression. Burgess et al. [17] demonstrated that lowly expressed ectopic Chfr is localised in the cytoplasm, but localises to the spindle during mitosis. They also 
showed that Chfr interacts with TCTP throughout the cell cycle, unless cells are treated with microtubule-destabilising agents. Two separate reports by Johansson et al. [22, 23] describe the interaction of TCTP with the two nuclear proteins, nucleophosmin and nucleolin, in embryonic stem cells. In the case of nucleophosmin, the interaction was shown to be independent of phosphorylation by Plk-1.

A recent paper unveiled a novel pathway, through which TCTP is likely to be involved in the dysregulation of mitotic progression in hepatocellular cancer, HCC [24]. The authors identified the TCTP gene, tpt1, as a target for transcriptional activation by CHD1L, a specific oncogene frequently upregulated in HCC. Overexpression of TCTP promoted the ubiquitin-proteasome degradation of the phosphatase Cdc25C. During mitotic progression, Cdc25C is essential for the final activation of Cdk1. The drop of Cdk1 activity induced by TCTP overexpression resulted in a too fast mitotic exit and consequently led to chromosome miss-segregation. The effect of TCTP overexpression on mitotic progression in tumour cells described here are consistent with earlier papers reporting that over- or underexpression of TCTP affects the duration of the cell cycle $[14,23,25]$. The observation that TCTP is one of the proteins ubiquitinylated and partially degraded in proteasomes during mitosis [18] would also indicate that tight regulation of TCTP levels is essential for the orderly exit of cells from mitosis.

It is well documented from plants [25, 26], as well as from lower animals, such as cnidarians [27], that TCTP is preferentially expressed in meristmatic/proliferative active tissues, and recently, a thorough study described TCTP as a general mitotic regulator in plants and animals [25]. This group demonstrated that TCTPs are interchangeable between plants (Arabidopsis) and animals (Drosophila) in their ability to rescue mitotic defects in the other species.

\section{- Importance of TCTP in Early Development}

The involvement of TCTP in mitotic regulation indicates that it could play a role in the early development of animals and plants. There are several lines of evidence clearly supporting this view:

1. Complete gene knockout of TCTP in Drosophila [28] and in mice [29-31] is embryonic lethal, and this effect was related to excessive apoptosis during early embryonic development of these mice. Koide et al. [31] provided data showing that TCTP functions as an inhibitor of the BMP pathway in the early phases of development.

2. In 2007, two papers reported a role for TCTP in the reprogramming of somatic cell nuclei to an embryolike gene expression pattern after transplantation into oocytes [32,33]. In this process, the transcription factor oct4, which is also considered a stem cell marker, is massively up-regulated. Koziol et al. [32] identified TCTP as one of the proteins that bind to the regulatory region of the mouse oct 4 gene and that activates its expression and hence the activation of genes involved in pluripotency. As mentioned in the previous section, TCTP was described as interaction partner for nucleolin [22]. This paper also demonstrated that nucleolin interacts with the transcription factor oct4 in human and murine embryonic stem cells.

3. Several reports indicate that TCTP plays a crucial role in early gametogenesis. Guillaume et al. [34] found high expression levels of TCTP in most stages of spermatogenesis, and Vitale et al. [35] showed that TCTP expression is altered during murine oocyte maturation. Meyvis and coworkers [36] reported that knock-down of TCTP in C. elegans reduced the numbers of eggs laid by the hermaphrodite in the $\mathrm{F}(0)$ and $\mathrm{F}(1)$ generations, indicating a pivotal role of TCTP in egg production.

4. A critical process in early mammalian development is the implantation of the fertilised egg. A recent publication reports that TCTP mRNA and protein levels increase in the uterus of mice during early pregnancy, reaching a peak at D5, but then decrease dramatically to D7 [37]. The peak of TCTP expression coincided with the implantation phase, and at this time TCTP levels were considerably lower in the uteri of pseudopregnant control mice. The number of implanted embryos was reduced when TCTP levels were downregulated by injection of antisense oligos. Arcuri et al. [38] described a role for TCTP in $\mathrm{Ca}^{++}$-handling and transport in the placenta. TCTP knock-down was associated with reduced cellular calcium-uptake and buffering capacity.

5. In plants, knockout of TCTP leads to a male gametophytic phenotype, with normal pollen formation and germination, but impaired pollen tube growth [26]. Silencing of TCTP by RNA interference slowed vegetative growth, and leaf expansion was reduced because of a smaller cell size. These data indicate that TCTP is also important for plant cell growth and development.

\section{Part of the Cell's Survival Kit: The Anti-Apoptotic Protein TCTP}

\section{- Protection Against Diverse Cell Stresses}

TCTP was originally described as a growth-induced protein (see section on TCTP and growth regulation below), and later it became clear that it is also highly regulated in response to a range of additional cellular stimuli and stress conditions. Examples of stress-dependent regulation of TCTP levels will be detailed in the regulation section below. Here, I will summarise the various mechanisms in discussion to-date, by which TCTP may exert its cyto-protective function.

\section{- A Role for TCTP in Ion-Homeostasis: Regulation of the $\mathrm{Na}^{+}-\mathrm{K}^{+}$-ATPase}

A potential role of TCTP in the regulation cellular ion homeostasis was revealed by Kyunglim Lee's group in Seoul. Using a yeast two-hybrid screen, they established that TCTP interacts with the $3^{\text {rd }}$ large cytoplasmic domain of two isoforms of the $\mathrm{Na}^{+}-\mathrm{K}^{+}$-ATPase [39]. They also showed that overexpression of TCTP resulted in inhibition of the enzyme. The $\mathrm{Na}^{+}-\mathrm{K}^{+}$-ATPase had been implicated in the pathogenesis of hypertension, and the authors hypothesised that overexpression of TCTP might promote the development of hypertension. They generated TCTP overexpressing 
mice, which indeed developed systemic arterial hypertension, about six weeks after birth [40].

\section{- Anti-Apoptotic Properties of TCTP (Fortilin)}

The first demonstration of the anti-apoptotic function of TCTP came from Fujise's laboratory in Houston [7]. Importantly, these authors demonstrated that overexpression of TCTP protected HeLa cells from undergoing etoposideinduced apoptosis. Based on their observations, they coined the name 'fortilin' for this protein and since refer to it under this name. Anti-apoptotic activity of TCTP has since been reported in numerous papers and for a range of cellular conditions. Here, I will give a few of the recently published examples:

Gnanasekar et al. [41] showed that human TCTP and its homologue from the parasite Schistosoma mansoni may act as a molecular chaperone under heat shock conditions, protecting other proteins against thermal denaturation. Several groups reported regulation of TCTP levels under $\mathrm{Ca}^{++}$-stress conditions [42-44], and overexpression of TCTP partially protected cells from induction of apoptosis by $\mathrm{Ca}^{++}$-stress $[12,42]$. The importance of TCTP as a survival factor, involved in the development of cellular resistance against anticancer drugs was demonstrated earlier for etoposide [7] and 5-fluorouracil [45].

The role of TCTP in protecting cells against oxidative stress was demonstrated through cloning of genes that confer such protection to NIH-3T3 cells [46]. Gnanasekar et al. [47] reported that TCTP of the parasite Brugia malayi functions as an antioxidant protein. More recently, Lucibello et al. [48] studied the importance of TCTP in the protection of cancer cells against oxidative stress, and showed that it is an important survival protein likely to be involved in the resistance against anti-cancer therapy. Interestingly, this paper also reports that glucose deprivation resulted in down-regulation of TCTP, followed by cell death. The latter finding is corroborated by our recent study on pancreatic beta-cells, showing that an exposure to high glucose $(25 \mathrm{mM})$ leads to an increase in intracellular TCTP levels [43]. This paper also demonstrated that overexpression of TCTP partially protects beta-cells against the induction of apoptosis by high levels of saturated fatty acids (palmitate), a particular stress situation for these cells.

The role of TCTP as an anti-apoptotic protein was highlighted in TCTP gene-knockout studies on mice [29-31], which demonstrated embryonic lethality due to excessive apoptosis at an early embryonic state. In 2009 two papers showed the importance of TCTP in preventing apoptotic cell death for the maintenance and development of T-cells. Using conditional TCTP knockout mouse models, Wu and colleagues [49] demonstrated that TCTP is essential for the viability of mature peripheral T-cells. Xiong et al. [50] reported that IL-2 promotes the survival of regulatory T-cells through stimulation of TCTP expression.

\section{- Mechanisms of Anti-Apoptotic Activity}

TCTP has been shown to interact with two other antiapoptotic proteins of the Bcl-2 family, i.e. Mcl-1 [51, 52] and Bcl-XL [53]. Bcl-XL and TCTP have both been implicated in maintaining the survival of murine T-cells during activation [53]. Two reports indicate that Mcl-1 and TCTP stabilise each other, with TCTP being stabilised by Mcl-1 [52] but also vice versa [51]. Yet another paper showed that they exert their anti-apoptotic function independent of each other [45].

A range of mechanisms have been proposed as to how TCTP exerts its anti-apoptotic activity. Graidist et al. [12] demonstrated a role of TCTP as a $\mathrm{Ca}^{++}$-scavenger, preventing excessive increase in intracellular $\mathrm{Ca}^{++}$-levels in $\mathrm{Ca}^{++}$stress conditions. Gnanasekar and colleagues reported that TCTP acts as molecular chaperone under heat shock conditions [41]. Telerman's group [30] proposed a novel mechanism, according to which TCTP, with its helical domain, inserts itself into the mitochondrial membrane, thereby antagonising the dimerisation of the pro-apoptotic protein Bax and preventing the Bax-induced increase of mitochondrial membrane permeability. A cell stress-dependent association of TCTP with mitochondria has been previously observed in yeast [54].

A peculiar involvement of TCTP in anti-apoptotic intercellular signalling was recently proposed by Sirois and coworkers [55]. This paper reports that TCTP is one of the marker proteins present at the surface of the nanovesicles released from apoptotic endothelial cells. These nanovesicles, which are different from apoptotic blebs, induce an anti-apoptotic phenotype in vascular smooth muscle cells (VSMC). Pre-treatment of endothelial cells with TCTP siRNAs attenuated the anti-apoptotic activity of purified nanovesicles on VSMCs.

\section{- Antagonism Between TCTP and P53}

Antagonism to the tumour suppressor protein p53 has recently been proposed as an another mechanism by which TCTP can prevent apoptotic cell death. It is not surprising, that activation of $\mathrm{p} 53$, one of the most powerful proapoptotic proteins, down-regulates the anti-apoptotic protein TCTP. Such observations have been made earlier, using a temperature sensitive mutant of p53, in two quite different cellular systems $[42,56]$. Several recent studies now describe TCTP as an interaction partner and antagonist for p53, albeit by differing mechanisms. Rho et al. [57] showed that overexpression of TCTP in lung carcinoma cells reversed p53mediated apoptosis, whereas TCTP knock-down increased apoptosis. Similarly, Chen and colleagues [58] observed TCTP (fortilin) interaction with p53 using GST-pull down assays; they showed that TCTP inhibits p53-dependent apoptosis. However, these two studies arrive at differing conclusions in the following two points: 1 . In the mapping of the domain in TCTP that is involved in p53 binding: Rho et al. [57] found that an internal domain (corresponding to the $\alpha$ helical domain; Fig. 1) is involved in p53 binding, whereas Chen et al. [58] observed that the $\mathrm{N}$ - and C-terminal parts of the molecule (the $\beta$-sheet domain; Fig. 1) participate in this interaction. 2. In the mechanism by which TCTP antagonises p53: Rho et al. reported that TCTP is destabilising p53 in lung cancer cells, whereas Chen and colleagues did not observe p53 destabilisation, but instead proposed that TCTP blocks p53-induced transcriptional activation of Bax [58].

A profound study on this issue has just been published by the Telerman group [59]. They describe details of a negative 
feedback loop between P53 and TCTP, according to which TCTP promotes MDM2-mediated ubiquitination and degradation of P53. Conversely, P53 directly represses TCTP transcription. This paper also provides evidence for the implication of this antagonism to P53 in cancer, which will be discussed below.

\section{Involvement of TCTP with Growth Regulation and the Translational Machinery}

\section{- TCTP and Cell Growth Regulation}

Translational induction of TCTP synthesis upon growth stimulation of mammalian cells was one of the earliest observations about this protein $[1,3]$. We typically observed an about four-fold increase of TCTP levels after growth induction of mouse fibroblasts [60]. Growth-dependent regulation of TCTP synthesis has been observed by many groups, as reviewed in our earlier article [8]. In the last decade, an overwhelming body of evidence has associated TCTP with cancer, as will be discussed below. A detailed study on TCTP in Arabidopsis thaliana demonstrated that knockdown of this protein generated serious growth defects [26], highlighting the importance of TCTP as a growth regulator, also in plants.

All these observations reflect a positive association with cell growth, however the question remains unanswered: What is the underlying mechanism, by which TCTP positively affects cell growth? It is attractive to speculate that TCTP's involvement in mitotic regulation might be important. However, we observed that overexpression of TCTP actually slows cell cycle progression [14], and very recently Chan et al. [24] showed that increased TCTP induces mitotic defects and chromosome miss-segregation in hepatocellular carcinoma. Thus, it appears that controlled expression of TCTP is essential for an orderly transition through the cell cycle, making it unlikely that mass induction of TCTP levels are required for progression through mitosis.

Two studies reported that an increase in TCTP levels results in activation of cell growth signalling pathways: Kim et al. [61] found that in HeLa cells, TCTP overexpression resulted in activation of several growth signalling pathways, inclusive of tyrosine phosphorylation of the EGF receptor, phosphorylation of PLC- $\gamma$, as well as activation of the Ras/Raf/ERK and the PI3K/Akt pathways. Specific inhibition of the PI3K/Akt pathway significantly decreased TCTP overexpression-induced cell survival, whereas inhibition of PLC- $\gamma$ pathway diminished TCTP overexpression-induced cell migration. In a more recent study, the same group performed similar experiments on human breast epithelial cells [62]. They found that TCTP induces release of the protein kinase Src from the $\mathrm{Na}^{+}-\mathrm{K}^{+}$-ATPase. Src activation then resulted in tyrosine phosphorylation of epidermal growth factor receptor and the activation of a range of signalling pathways, such as the PI3K/Akt, the MAP kinase, and the PLC- $\gamma$ pathways, apart from other cellular alterations. Conversely, Wang and Dao [63] reported that TCTP is down-regulated during neural differentiation of mouse embryonic stem cells.

The regenerating rat liver after partial hepatecomy is a classical model for tissue growth. Zhu et al. [64] studied TCTP mRNA expression in this model and demonstrated that TCTP mRNA levels were significantly up-regulated in the period from 3 to 12 hours after partial hepatectomy, but then decreased at 24 hours before returning to original levels.

Cell growth is intimately linked with an increase in protein synthesis, and early observations demonstrated that both protein synthesis and ribosome biogenesis are largely increased after partial hepatectomy. Several other observations also potentially link TCTP with the translational machinery and its regulation; these will be discussed in the following section.

\section{- Is TCTP an Upstream Regulator of the mTOR Signal- ling Pathway?}

The mammalian target of rapamycin complex 1 (mTORC1) signalling pathway is a major regulator of protein synthesis in mammalian cells [65]. This pathway represents a complex signalling network, which integrates a range of positive and negative input signals and positively regulates protein synthesis, as well as other anabolic pathways. Most relevant for the regulation of protein synthesis is the regulation of $\mathrm{mTORC} 1$ in response to growth factor signalling, to amino acid availability and to the energy status of the cell.

An important upstream activator of mTORC1 is the small GTPase Rheb (Ras homologue enriched in brain), which in turn is negatively regulated by its GTPase-activating protein, the tumour suppressor protein TSC1/TSC2. The guanosine nucleotide exchange factor (GEF) for Rheb remained for long time (and still is) enigmatic. In 2007, Hsu et al. [28] published a paper describing TCTP as a potential GEF for Rheb. They presented data indicating that reducing Drosophila TCTP (dTCTP) levels reduced cell size, cell number and organ size, resembling dRheb mutant phenotypes. They also found that dTCTP directly associates with dRheb and can act as a GEF for Rheb. Thus, this paper ascribed an important role to TCTP, as a positive regulator of the TOR growth signalling pathway.

The idea that TCTP acts as a GEF for small GTPases, such as Rheb, appeared attractive based on TCTP's structural similarity to MSS4/DSS4 proteins, which are known to bind to the Rab family of GTPases [10]. However, subsequent investigations aimed at confirming TCTP's importance in regulating $\mathrm{mTORC} 1$ signalling in mammalian cells failed to support the findings published for Drosophila TCTP: We observed that reducing TCTP levels did not reproducibly affect mTORC1 signalling in HEK 293 cells [66]. Moreover, overexpression of TCTP did not rescue mTORC1 signalling in amino acid-starved cells, and no stable interaction between TCTP and Rheb or mTORC1 was observed. Similarly, Rehmann et al. [67] were unable to detect GDP exchange activity of TCTP towards Rheb. TCTP depletion in cells did not affect direct downstream targets of Rheb, and no interaction between TCTP and Rheb could be detected by NMR spectroscopy. However, another study reported a weak binding between Rheb and TCTP, and also a GEF activity for TCTP towards Rheb [68]. These authors also performed further structure modelling in support of the interaction. Overall, this issue is still controversial. We are aware, from personal communications, that other laboratories also obtained negative results in this regard, which have not been 
Table 1. TCTP Expression in Cancer Cell Lines

\begin{tabular}{|c|c|c|c|}
\hline Cells Derived From & Cell Lines & Result Regarding TCTP & Reference \\
\hline Colon cancer & SNU-C4, C5 & mRNA levels $2-4.5$ fold increased compared to normal cells & [109] \\
\hline Colon cancer & LoVo-cells & $\begin{array}{l}\text { TCTP knock-down results in decreased cell proliferation, migration, } \\
\text { invasion and tumorigenicity. }\end{array}$ & [81] \\
\hline Prostate cancer & LNCaP & TCTP knock-down results in decreased cell viability and increased apoptosis & [79] \\
\hline Bronchial epithelia (transformed) & 1170-I, 1198, & \multirow{2}{*}{ TCTP secretion is two-fold higher in transformed $v s$. non-transformed cells. } & \multirow{2}{*}{ [75] } \\
\hline (non-transformed) & BEAS-2B, 1799 & & \\
\hline Human leukaemia & U937, K562 & \multirow{2}{*}{$\begin{array}{l}\text { Reduction of TCTP levels leads to reduced tumorigenicity in mice } \\
\text { and reversion from the malignant phenotype. }\end{array}$} & \multirow[b]{2}{*}{ [56] } \\
\hline Breast carcinoma & $\begin{array}{l}\text { MDA-MB231S } \\
\text { BT20, T47D }\end{array}$ & & \\
\hline $\begin{array}{l}\text { Colon cancer } \\
\text { Lung cancer } \\
\text { Melanoma }\end{array}$ & $\begin{array}{l}\text { DLD-1 } \\
\text { A549 } \\
\text { various lines }\end{array}$ & \multirow[t]{2}{*}{$\begin{array}{l}\text { Cell clones reverted from the transformed to the normal phenotype display } \\
\text { lower TCTP expression. }\end{array}$} & [77] \\
\hline Multiple Myeloma & RPMI8226 & & [78] \\
\hline
\end{tabular}

published. Therefore, currently this potentially interesting function of TCTP remains an unresolved question.

\section{- Is TCTP Part of the Translational Apparatus and a Downstream Target of mTOR?}

The mRNA for TCTP starts with a 5'-terminal oligopyrimidine tract (5'-TOP) [69]. This observation suggests that TCTP expression may be subject to translational control via the mTORC1 signalling pathway. Almost all mRNAs that bear this signature encode proteins that are part of the translational apparatus, and they are known to be translationally up-regulated through mTORC1 [65]. It therefore seems likely that TCTP mRNA is a downstream target of mTOR (see TCTP regulation; below).

Direct evidence for a role of TCTP in the translational machinery came from a study by Cans et al. [70]. Using a yeast two-hybrid search, they identified TCTP as a binding partner for protein synthesis elongation factor eEF1A, and its guanine nucleotide exchange factor, eEF1B $\beta$. The interaction of TCTP with eEF1B $\beta$ was also confirmed by Langdon et al. [71]. Cans and colleagues demonstrated that TCTP preferentially stabilizes the inactive GDP-bound form of eEF1A, by impairing the GDP exchange reaction promoted by eEF1B $\beta$. From this study, it would appear that TCTP acts as a negative regulator for eEF1A, which is apparently inconsistent with a growth-promoting role of TCTP.

A more recent report provided an interesting twist to this story: Leclerq et al. [72] performed a study on Sphingosine kinase 1 (SK1), which catalyses the formation of the phospholipid sphingosine 1-phosphate. Elevated activity of this enzyme enhances cell proliferation and survival, and is implicated in tumorigenesis. This group had previously shown that eEF1A interacts with and activates SK1. In this study, they demonstrate that it is [eEF1A x GDP], but not [eEF1A $x$ GTP], that activates SK1 activity in vitro. They also show that enhancing cellular [eEF1 x GDP] levels through expression of TCTP (which acts here as guanine nucleotide dissociation inhibitor of eEF1A) increases cellular SK1 activity. In this way, TCTP could be implicated in tumorigenesis, even though being a negative effector of protein synthesis.
More work is required to determine, which potential role TCTP might play in protein synthesis.

\section{Is TCTP a Tumour Protein? \\ - TCTP Expression in Human Tumours}

The observation that TCTP is expressed in nearly all cell types and tissues, albeit at differing levels [73] initially raised some doubts on the justification of the term 'tumour protein' [74]. However, over the past decade, a substantial body of evidence has accumulated, demonstrating that TCTP is positively related to cancer: First, several studies on cancer cell lines, derived from a range of cancer types, have shown that TCTP levels are positively related to properties of these cells relating to growth behaviour, anti-apoptotic properties and tumorigenicity (Table 1). Second, studies on several human cancers have demonstrated that TCTP levels are upregulated in most, but not all, of the investigated cancer types (Table 2). In some cases, such as lung cancer [75], hepatocellular carcinoma [24] and breast cancer [59], TCTP has even been proposed to be suitable as a tumour marker. Our recent data obtained by immunohistostaining of human colon cancer samples also indicate that TCTP levels are significantly increased in adenomas and adenocarcinomas of the colon, compared to normal colon tissue (M. Radojkovic, P. Colligan, P. Puri, A. Lochhead, M. Aghmesheh \& U. Bommer, unpublished results).

\section{- TCTP in the Tumour Reversion Model}

The importance of TCTP in cancer has been convincingly demonstrated through studies using the tumour reversion model. This model is based on the rare event of tumour cells reverting back from the malignant to the normal phenotype (reviewed in [76]). Telerman's group studied this system intensively and identified a number of genes that are particularly involved in this process, of which TCTP appears to play a major role. In particular, they showed that inhibition of TCTP expression by siRNA results in suppression of the malignant phenotype [56]. They also demonstrated: (1.) that revertants derived from melanoma cell lines, colon or lung cancer show decreased TCTP levels; (2.) that inhibition of TCTP expression by antisense DNA led to a 'flat rever- 
Table 2. TCTP Levels in Human Cancers

\begin{tabular}{|l|l|l|l|}
\hline \multicolumn{1}{|c|}{ Cancer Types } & \multicolumn{1}{|c|}{ TCTP Levels } & Method/Comment & Reference \\
\hline \hline $\begin{array}{l}\text { Colorectal and lung cancer; } \\
\text { Oesophageal, hepatocellular, pancreatic cancer }\end{array}$ & $\begin{array}{l}\text { up-regulated } \\
\text { not altered }\end{array}$ & $\begin{array}{l}\text { Proteomics studies on tissues and } \\
\text { body fluids }\end{array}$ & [110] \\
\hline $\begin{array}{l}\text { Six Lung SSCs vs. normal tissue; } \\
\text { Five Lung adenocarcinomas }\end{array}$ & usually up-regulated & Western blot of cell lysates \\
\hline Serum of lung cancer patients vs. serum of healthy controls & about 2.3-fold up-regulated & Western blot; densitometry \\
\hline Breast cancer vs. surrounding tissue & up-regulated & Proteomics study \\
\hline Breast cancer & high TCTP associated with aggressive tumours; & Immunohistochemistry \\
\hline $\begin{array}{l}\text { Tumours of liver, lung, thyroid, larynx, } \\
\text { pkin, uterus, breast, ovary, prostate, rectum, } \\
\text { Tumours of cervix, pancreas, stomach, testis }\end{array}$ & $\begin{array}{l}\text { up-regulated } \\
\text { not up-regulated }\end{array}$ & Western blot of cancer samples \\
\hline Liver cancer vs. adjacent normal tissue & up-regulated & [111] \\
\hline Hepatocellular carcinoma & up-regulated in most of the advanced tumours & [59] \\
\hline Colorectal cancer (20 tumour samples) & $\begin{array}{l}\text { 2.0-fold up-regulated } \\
1.2 \text {-fold up-regulated }\end{array}$ & $\begin{array}{l}\text { Immunohistochemistry } \\
\text { mRNA levels by microarray; } \\
\text { by real-time PCR }\end{array}$ \\
\hline
\end{tabular}

sion phenotype' of transformed NIH3T3 cells and (3.) that reduction of TCTP levels raised the number of spontaneous revertants dramatically [77]. These results were corroborated by a recent study using a proteomics approach to identify proteins altered in tumour reversion in multiple myeloma cells. This group reported that, among others, STAT3, TCTP, CDC2 and PCNA are down-regulated in the tumour reversion process [78].

\section{- Mechanisms Through which TCTP Could Promote Cancer}

The most obvious way, by which TCTP is likely to promote cancer, is through its anti-apoptotic activity. In analogy to many other anti-apoptotic proteins, such as Bcl-2, Mcl-1 or Bcl-XL, overexpression of TCTP is part of the armoury of cancer cells to evade apoptosis. For example in LNCaP prostate cancer cells, decreased expression of TCTP was associated with reduced cell viability [79]. Similarly, Lucibello et al. [48] demonstrated that cell clones with forced TCTP expression, derived from the breast cancer cell line MDA-MB231, displayed reduced sensitivity to oxidative stress, whereas cells with down-regulated TCTP showed increased sensitivity.

Another study also underscored the importance of preventing apoptosis for the role of TCTP in cancer. Lee et al. [80] established that TCTP (fortilin) interacts with transforming growth factor-beta stimulated clone-22 (TSC-22). Overexpression of TCTP in ovarian carcinoma cells resulted in increased degradation of TSC-22 and the reversal of TSC22-mediated apoptosis, whereas knockdown of TCTP led to an increase in apoptosis in these cells.

The antagonism between TCTP and the tumour suppressor protein P53 is likely to play a considerable role in TCTP's ability to promote cancer. Substantial evidence for this conclusion has been provided in the recently published paper by Amson et al. [59]. These authors found that in about 500 breast cancer patients, a high-TCTP status is associated with aggressive tumours, and predicts a poor progno- sis. Also, TCTP knockdown in primary mammary tumour cells from ErbB2 transgenic mice resulted in increased P53 expression and a decreased number of stem cell-like cancer cells.

Ma et al. [81] investigated the effect of TCTP knockdown on proliferation, migration, and invasion properties of colon adenocarcinoma cells. They used 2D gel electrophoresis to identify proteins, whose expression levels are altered after TCTP knockdown and found that components of the ubiquitin-proteasome system, proteins involved in the biosynthesis of cytoskeletal proteins and in tumour metastasis were altered upon TCTP removal.

Another recent study, on human hepatocellular carcinoma cells [24], proposed a different mechanism of TCTPdependent tumorigenesis. These authors studied the mode of action of the chromodomain helicase DNA binding protein 1like (CHD1L), which is amplified and acts a specific oncogene in $>50 \%$ of human hepatocellular carcinomas (HCC) and found that CHD1L targets the transcription of the TCTP gene tpt1. Overexpression of TCTP was found in about $41 \%$ of human HCC samples, and it highly correlated with the advanced tumour stage of HCC patients. Further investigations revealed that the tumorigenicity of TCTP is linked to a role in mitotic regulation, as described earlier. TCTP induces a faster mitotic exit and chromosome miss-segregation, resulting in chromosome instability.

Thus, these two recently published studies describe two important, but quite different mechanisms of TCTPdependent cancer development. Amson et al. [59] emphasise the importance of TCTP-dependent P53 degradation using the example of breast cancer, whereas Chan et al. [24], for hepatocellular carcinoma, describe a novel mode of action of TCTP, based on its role in mitotic cell cycle regulation. A common underlying mechanism appears to be the promotion by TCTP of the ubiquitin-proteasome degradation of proteins crucial for cellular homeostasis or cell cycle progression. In this context, the finding by Ma et al. [81] is interest- 
ing, that after knock-down of TCTP the expression of components of the ubiquitin-proteasome system is altered.

\section{- Is TCTP Suitable as an Anti-Cancer Drug Target?}

The multiple roles/mechanisms described so far for TCTP-dependent tumorigenicity provoke the question of whether TCTP is or can be made a suitable drug target in anti-cancer treatment. Whilst, to my knowledge, no report directly addressing this question has been published as yet, there are a couple of papers touching on this important point. In model studies on prostate cancer cells Gnanasekar et al. [79] showed that transfection with TCTP siRNA reduced the viability of these cells. Yao et al. [82] performed a comparative proteomic analysis to survey changes in protein expression levels after treatment of three colon cancer cell lines with the anti-cancer drug oxaliplatin. TCTP was among the 21 proteins found to be altered in all three cell lines. Its expression was significantly up-regulated within $24 \mathrm{~h}$, but then gradually decreased from 48 to $72 \mathrm{~h}$. Tuynder and colleagues [77] tested a panel of drugs for their ability to suppress TCTP protein levels in U937 cells, and it appeared that this ability correlated well with the potency of these drugs to induce cytotoxicity in these cells. Taken together, these observations would suggest that TCTP might be suitable as a biomarker in testing the drug efficacy in cellular anti-cancer drug testing.

The suitability of TCTP as direct anti-cancer drug target has been reported for three drugs. Since the earlier discovery of Plasmodium TCTP as a potential target for the antimalarial drug artemisinin [83], the interaction of TCTP with this drug has been investigated in more detail [84]. Artemisinin is also considered as an anti-cancer drug [85], and Fujita et al. [86] published a study on the interaction of human TCTP (fortilin) with dehydroartemisinin (DHA). They demonstrated that DHA binds to human TCTP and decreases cellular TCTP levels through promoting ubiquitination and proteasome-dependent degradation. In their recent article on the antagonism between TCTP and P53, Amson et al. [59] report the targeting of TCTP by the pharmacological compounds sertraline and thioridazine, which interfere with the down-regulation of P53 by TCTP.

Taken together, the wide range of observations summarised in this section, provides a solid justification for the term 'tumour protein' in the name of TCTP.

\section{REGULATION OF CELLULAR TCTP LEVELS}

\section{Cell Physiological Conditions Resulting in Regulation of TCTP Levels}

There is a wealth of publications reporting changes of TCTP levels in response to alterations of cell physiological conditions. Many of the groups now working on TCTP discovered this protein through such type of observations. It would be beyond the scope of this article, to comprehensively review all these reports. This section will briefly summarise the main types of extracellular signals that lead to regulation of TCTP levels.

\section{- Growth Signals and Nutrients}

As pointed out above, the induction of TCTP synthesis by cell growth signals is well documented (reviewed in [8]).
Many of these studies used growth stimulation of serumstarved cells and foetal calf serum as general source of growth factors, whereas only few studies investigated the potential of individual growth factors to induce TCTP synthesis. Vercoutter-Edouart et al. [87] demonstrated that in MCF-7 human breast cancer cells, TCTP was among four proteins up-regulated by fibroblast growth factor- 2 . Recently, we found that TCTP levels are increased about twofold after insulin treatment in HT29 colon cancer cells (J. Chen \& U.A. Bommer, unpublished observation). Schmidt et al. [88] demonstrated that TCTP synthesis is induced by phorbol ester and forskolin, both at the transcriptional and posttranscriptional levels of gene expression, and two other papers reported the regulation of TCTP levels through the vitamin D receptor pathway $[89,90]$.

There is only sparse information about nutrient regulation of TCTP levels. Back in 2000, Bonnet et al. reported downregulation of TCTP (together with ribosomal protein S6) upon ammonium starvation in fission yeast. However, it is likely that this treatment merely reflects a stress condition rather that a genuine nutritional signal. Our recent study on pancreatic beta-cells demonstrated that TCTP levels are subject to glucose regulation in this cell type [43].

\section{- Cell Stress Conditions}

The induction of TCTP in response to various stresses was initially reported more than ten years ago, for mammalian cells [44, 91], as well as for worms [92] and for plants [93]. The types of stresses known to date that result in TCTP induction and/or down-regulation include the following groups:

1. Heat shock. Induction of TCTP synthesis under heat stress conditions was originally noted in parasitic organisms, such as Trichinella [94,95] and Schistosoma [41]. A recent study investigated the importance of TCTP for stress tolerance of the cabbage, Brassica oleracea, and found that silencing of the TCTP gene by RNAi led to reduced vegetative growth rate and decreased tolerance of cold, high temperature and salt stresses by the cabbage plants [96].

2. Oxidative stress. The first study reporting an oxidation status-dependent regulation of the TCTP gene was published by Rupec et al. [91]. These authors set out to clone genes that are induced under hypoxia, as it occurs in solid tumours. They observed a 12-fold up-regulation of a form of TCTP (P23) mRNA, fused to the mitochondrial $16 \mathrm{~S}$ ribosomal RNA under conditions of hypoxia. They also found that hypoxia induced up-regulation of TCTP mRNA and 16S RNA individually in HeLa cells.

Yan et al. [97] investigated the oxidative stress response in a non-malignant breast epithelial cell line and in a derived malignant cell line. TCTP (fortilin) was found to be significantly up-regulated after hydrogen peroxide treatment in the transformed cells, but not in the parental cells. Gnanasekar and colleagues [47] reported that TCTP from the filarial parasite Brugia malayi has anti-oxidant properties. Nagano-Ito et al. [46] found that expression of TCTP 
cDNA, confers resistance to hydrogen peroxide treatment to mouse NIH-3T3 fibroblasts. Rid and coworkers observed that in human keratinocytes, under mild oxidative stress, TCTP translocates into the nucleus [90].

A very recent study by Lucibello et al. [48] showed that in the breast cancer cell line MDA-MB-231 the sensitivity to oxidative stress was strongly enhanced in cells with reduced TCTP levels, and decreased in cells with high TCTP levels. The authors propose TCTP as a 'stress hallmark' in cancer cells. The same paper also investigated the regulation of TCTP under oxidative stress in a range of tumour cell lines. Depending on the severity of the cell stress, the authors found either up- or down-regulation of TCTP levels: In mild oxidative stress, TCTP was upregulated in cells that survived the treatment. In contrast, severe oxidative stress caused downregulation of TCTP followed by cell death. Generalising from these observations it would seem that under mild stress conditions, TCTP is induced as part of the cell's defence system, whereas under severe stress, pro-apoptotic mechanisms prevail, which include the down-regulation of anti-apoptotic

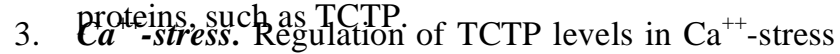
conditions has initially been studied by $\mathrm{Xu}$ et al. [44] in COS-7 cells. They observed an increase in TCTP levels under these conditions, regulated at both transcriptional and post-transcriptional levels. In contrast, in our later study on mouse embryo fibroblasts we found that TCTP levels are down-regulated under calcium ionophore or thapsigargin treatment [42]. Interestingly, in some cases, we also saw a moderate initial up-regulation of TCTP levels, followed by a more substantial down-regulation upon prolonged treatment. The conclusion drawn in the previous paragraph from the paper by Lucibello et al. [48] would be consistent with this latter observation, and it could provide an explanation to reconcile the two apparently contradicting results for TCTP regulation in $\mathrm{Ca}^{++}$stress $[42,44]$. Decrease of TCTP levels were also observed in pancreatic beta-cells upon stress induced by treatment with thapsigargin or saturated fatty acids, i.e. palmitate [43].

4. Stress induced by heavy metals. The most dramatic alterations of TCTP levels have been observed under exposure of cells or organisms to heavy metals. The first paper reporting such an example investigated the gene expression pattern of earthworms (Lumbricus rubellus) exposed to heavy metal-infested soils from the mining industry. It reported an at least four-fold increase in TCTP (mRNA) levels in a earthworm population from a $\mathrm{Pb} / \mathrm{Zn} / \mathrm{Cd}$-polluted mine and an even 335-fold increase in earthworms from a $\mathrm{Cu}$ polluted mine [92]. Schmidt et al. [88] working with two mammalian cell lines, found that cobalt and nickel induced TCTP expression about two- to threefold at the mRNA and protein levels, whereas copper resulted in a five-fold induction of TCTP mRNA or protein levels. TCTP was also identified as one of the proteins specifically expressed in plants that are tolerant to aluminium-stress [93].

5. Stress induced by toxins or drugs. Oikawa et al. [98] studied the effect of tetrachlorodibenzo-p-dioxin (TCDD, or 'dioxin') on the mRNA expression patterns of mouse embryonic stem cells. The mRNA of TCTP (HRF) was found to be up-regulated by TCDD, which also induced the synthesis and secretion of TCTP. Another group of toxic and carcinogenic pollutants are the polycyclic aromatic hydrocarbons (PAHs). Zheng et al. [99] investigated the effect of PAHs in soil on the expression of TCTP in the earthworm Eisenia fetida and found that it was upregulated by benzopyrene, but down-regulated by phenanthrene. The effect of anti-cancer drugs on TCTP levels was discussed earlier.

\section{Mechanisms Involved in Regulation of Cellular TCTP Levels}

Whilst TCTP was originally discovered as a translationally regulated protein, it is now overwhelmingly clear that regulation of cellular TCTP levels may occur at several levels of gene expression and through a range of mechanisms. This is not surprising, given the variety of stresses or stimulatory signals that have been described to regulate TCTP levels, and considering the velocity and extent of alteration of cellular TCTP levels. Moreover, this is well in line with observations published for other anti-apoptotic or stress proteins, which are also regulated at multiple levels. Here, I will focus on the major mechanisms known to-date to be involved in TCTP regulation.

\section{- Gene Regulation at the Transcriptional Level}

The most detailed studies on gene structure and transcriptional regulation of TCTP synthesis were published by the Thiele laboratory in Berlin. Initially, they described the gene structure and upstream promoter sequences of the TCTP gene from rabbit and humans and established that these regions contain several promoter elements that are wellconserved in mammals. They also showed that, as a whole, this upstream region exhibits a strong promoter activity [100]. In a second report, the group characterised various features of TCTP mRNAs. Typically, cells generate two forms of this mRNA that differ in the length of their 3'untranslated regions. These were found to be transcribed in all rabbit and human tissues investigated, but at a considerable range of overall quantity and ratio of expression [73]. This paper also characterises four of the numerous processed, intronless pseudogenes of TCTP in the rabbit genome. This earlier work is summarised in our previous review article [8]. A similar paper, characterising the genomic structure and several pseudogenes of the mouse TCTP gene, was published at the same time by the Telerman group [101].

Additional evidence for transcriptional regulation of TCTP levels under specific physiological conditions came from other publications, such as Stuerzenbaum et al. [92], who found extreme differences in TCTP mRNA levels in earthworms exposed to soils polluted with specific heavy metals, as discussed above. Xu et al. [44] reported both transcriptional and translational regulation of TCTP levels under 
$\mathrm{Ca}^{++}$-stress situations in COS-7 cells. Bonnet et al. [102] studied genes that are specifically regulated under ammonium starvation in the fission yeast $S$. pombe, which leads to growth arrest and cell cycle exit. They found that the genes for ribosomal protein rpS6 and TCTP are repressed under this condition, and that their expression is co-ordinately regulated in many different growth conditions. Under ammonium starvation, transcriptional inhibition of these genes was regulated either by the PKA pathway and/or by the Wis1/MAP kinase pathway [102]. Oikawa et al. [98] showed that the dioxin-stimulated expression of TCTP (HRF) mRNA occurs via an aryl hydrocarbon receptor (AhR)dependent pathway.

These were the first reports on specific signalling pathways/transcription factors that are involved in transcriptional activation of the TCTP gene. A further study of the promoter region of the human TCTP gene, again by the Thiele group, revealed that transcriptional regulation of TCTP occurs by PKA signalling, via activation of members of the CREB family of transcription factors [103], which is consistent with the conclusions obtained from fission yeast mutants mentioned above [102]. Other transcription factors recently shown to directly regulate the transcription of the tpt- 1 (TCTP) gene include CHD1L [24] and the tumour suppressor protein $\mathrm{p} 53$ [59].

Another paper from the Thiele laboratory investigated both transcriptional and post-transcriptional regulation of the tpt-1 (TCTP) gene in mammalian cell lines under a variety of conditions known to result in alterations of TCTP levels [88]. Phorbol ester, forskolin, dioxin, cobalt, nickel and, to the highest extent, copper induced TCTP expression at both the mRNA and protein levels. The strong copper-dependent transcriptional activation was transmitted by a metalresponse element residing in the tpt-1 promoter. In contrast, the more moderate effects by cobalt and nickel where mediated by mRNA stabilisation, rather than by transcriptional activation [88].

\section{- Translational Regulation of TCTP Levels}

Early evidence for translational regulation of TCTP emerged from the following observations: 1. The seruminduced increase in the rate of TCTP (P23 or Q23) synthesis clearly preceded that of transcriptionally regulated proteins, and it was not inhibited by actinomycin $\mathrm{D}[1,3]$. 2. The mRNA of TCTP (P21) was found abundantly in untranslated cytoplasmic mRNP particles [2], which are presumed to be a cytoplasmic 'reserve pool' of untranslated mRNAs.

At that time, knowledge about translational control mechanisms was just beginning to emerge. In the early nineties, the importance of the cap-binding initiation factor eIF4E for the efficient translation of otherwise poorly translated mRNAs was just being appreciated. Such mRNAs typically code for proteins, which are involved in the cell cycle and cancer (reviewed in [104]). Since TCTP (P23) was described as growth-induced translationally controlled protein, we investigated whether it might be one of the mRNAs specifically regulated by eIF4E. We made use of eIF4E overexpressing fibroblasts to demonstrate that the rate of synthesis of TCTP is indeed correlated with eIF4E levels/activity in the cell [105].
Since this publication, important additional elements of the signalling pathways and mechanisms that regulate protein synthesis have been elucidated. It has become clear that the mammalian target of rapamycin complex 1 , mTORC1, is central to the complex regulatory network for protein synthesis and that eIF4E is an important element of this network, downstream of mTORC1. The eIF4E binding proteins (eIF4E-BPs), which normally inhibit eIF4E, are inactivated through phosphorylation by mTORC1 (reviewed in [65]).

There are two key features of the TCTP mRNA that make it a prime candidate for an mRNA specifically regulated by mTOR (and eIF4E): 1. As mentioned above, TCTP mRNA has a 5-terminal oligopyrimidine tract (5'-TOP), the signature typical for mRNAs coding for proteins that are part of the translational machinery and are known to be regulated by mTORC1. 2. We have previously shown that TCTP mRNA is a highly structured RNA molecule [60], and translation of such mRNAs is particularly dependent on the activity of eIF4E.

For these reasons, we are currently reinvestigating the mechanisms involved in growth-factor-dependent translational up-regulation of TCTP synthesis; in particular we address the question of the involvement of the mTORC1 pathway. We have now obtained results demonstrating that in HeLa cells, serum-induced synthesis of TCTP is partially inhibited by rapamycin, and completely inhibited by inhibitors of mTOR kinase activity and of the protein kinase Akt, which is upstream of mTORC1. In addition, eIF4E overexpression drives TCTP mRNA from the subpolysomal fraction into actively translating polysomes (U.A. Bommer, V. Iadevaia and C.G. Proud, unpublished results). These data indicate that TCTP mRNA behaves like a genuine 5'-TOP mRNA that is regulated by mTORC1.

Another key regulatory mechanism of translation, which leads to cell stress-dependent down-regulation of protein synthesis, involves the phosphorylation of the $\alpha$-subunit eukaryotic initiation factor eIF2. There are four distinct eIF2 $\alpha$ kinases, which are each activated under specific cell stress conditions. We have studied the effect of the doublestranded RNA-dependent protein kinase PKR on TCTP mRNA translation [60] and have shown that the highly structured TCTP mRNA is able to bind to and activate PKR, resulting in its own translational inhibition. We have also demonstrated that PKR is indeed necessary for the downregulation of TCTP synthesis under specific stress conditions, such as serum starvation [60] or $\mathrm{Ca}^{++}$-stress conditions [42].

The involvement of these two key regulatory mechanisms in the regulation of intracellular TCTP levels is in line with corresponding observations for other anti-apoptotic proteins. For example, it is well established that the synthesis of proteins like Bcl-XL, Mcl-1 and survivin are regulated through the mTORC1 pathway and require eIF4E activity for their translation [106]. Synthesis of Mcl-1 is also downregulated by PKR [107].

\section{- Other Post-Transcriptional Regulatory Mechanisms}

Schmidt et al. [88] investigated the mechanisms involved in the regulation of TCTP by heavy metals, and found that 
the moderate inductive effects by cobalt and nickel are mediated by mRNA stabilisation, whereas the strong inductive effect by copper was mediated both at transcriptional and posttranscriptional levels. They speculated that the AUUUA elements present in the 3'-untranslated region of TCTP mRNAs might be involved in the regulation of TCTP mRNA stability. However, these AUUUA elements do not completely match the features of canonical AU-rich elements (AREs) known to be involved in the destabilisation of cytokine mRNAs, and nobody has this far directly investigated their importance in TCTP mRNA. Generally, TCTP mRNA has been found to be fairly stable and abundant in mammalian cells, although the abundance may vary considerably, depending on the tissue type [73]. Thus, mRNA stability regulation as potentially effective regulatory mechanism for TCTP protein levels should be looked at in the context of the specific tissue and regulatory condition.

There are few reports describing the regulation of TCTP levels through protein degradation. Kubiak and colleagues [18] set out to characterise proteins that are specifically degraded in the first embryonic mitosis in Xenopus laevis. They identified TCTP as one of the proteins that are ubiquitinylated and potentially degraded via the proteasome in this phase of the cell cycle. Zhang et al. [52] described the interaction of TCTP (fortilin) with the anti-apoptotic protein Mcl1 and demonstrated in Mcl-1 knockdown experiments that TCTP is stabilised by Mcl-1. The same group reported later that dehydroartemisinin (DHA), an important anti-malarial drug, which was previously shown to bind to Plasmodium falciparum TCTP, binds to and destabilises human TCTP in a proteasome-dependent manner [86]. This is interesting, since DHA is also considered an anti-cancer agent $[85,108]$. Although the reports on regulated protein degradation of TCTP are limited and anecdotal so far, it appears that this type of regulation may occur in a distinct cell cycle phase and under pro-apoptotic cell stress conditions.

\section{CONCLUDING REMARKS}

After a slow beginning, research on the translationally controlled protein TCTP has accelerated considerably over the past 30 years, with a large number of groups having contributed many different facets to the 'TCTP story'. Functional importance has been attributed to this protein in a range of biological contexts, on both the molecular and the cellular levels. Since TCTP plays a major role in cell growth, division and reactions to stress situations, its cellular levels are subject to a considerable degree of regulation. It is therefore not surprising that TCTP regulation occurs at several levels of gene expression and engages a range of mechanisms. TCTP's importance for cellular homeostasis is further reflected at the organismic level, as obvious from its involvement in diseases processes, such as cancer or allergy. It will be important to better understand the molecular interactions and cellular roles of TCTP, and how they underpin such disease processes.

\section{CONFLICT OF INTEREST}

None declared.

\section{ACKNOWLEDGEMENTS}

Work in my laboratory was supported by grants from The Wellcome Trust, by a Short-Term Fellowship from the Human Frontier Science Program (Strasbourg), and by small grants from the Cancer Prevention Research Trust (London, UK), from the Graduate School of Medicine and the Illawarra Health and Medical Research Institute, University of Wollongong. I thank Professors Chris Proud (Southampton) and Thomas Preiss (Canberra) for helpful comments on parts of the manuscript.

\section{NOTE ADDED IN PROOF}

While this article was in press, a new paper was published, documenting a novel function for TCTP: Zhang J, de Toledo SM, Pandey BN, Guo G, Pain D, Hong Lie, and Azzam EI. Role of the translationally controlled tumor protein in DNA damage sensing and repair. Proc. Natl. Acad. Sci. USA 2012 (www.pnas.org/cgi/doi/10.1073/pnas.11063-00109 <http://www.pnas.org/cgi/doi/10.1073/pnas.1106300-109>).

\section{REFERENCES}

[1] Thomas G, Luther H. Transcriptional and translational control of cytoplasmic proteins after serum stimulation of quiescent Swiss 3 T3 cells. Proc Natl Acad Sci USA 1981; 78(9): 5712-6.

[2] Yenofsky R, Bergmann I, Brawerman G. Messenger RNA species partially in a repressed state in mouse sarcoma ascites cells. Proc Natl Acad Sci USA 1982; 79(19): 5876-80.

[3] Bohm H, Benndorf R, Gaestel M, et al. The growth-related protein P23 of the Ehrlich ascites tumor: translational control, cloning and primary structure. Biochem Int 1989; 19(2): 277-86.

[4] Chitpatima ST, Makrides S, Bandyopadhyay R, Brawerman G Nucleotide sequence of a major messenger RNA for a 21 kilodalton polypeptide that is under translational control in mouse tumor cells. Nucleic Acids Res 1988; 16(5): 2350.

[5] Gross B, Gaestel M, Bohm H, Bielka H. cDNA sequence coding for a translationally controlled human tumor protein. Nucleic Acids Res 1989; 17(20): 8367.

[6] MacDonald SM, Rafnar T, Langdon J, Lichtenstein LM. Molecular identification of an IgE-dependent histamine-releasing factor. Science 1995; 269(5224): 688-90.

[7] Li F, Zhang D, Fujise K. Characterization of fortilin, a novel antiapoptotic protein. J Biol Chem 2001; 276(50): 47542-9.

[8] Bommer UA, Thiele BJ. The translationally controlled tumour protein (TCTP). Int J Biochem Cell Biol 2004; 36(3): 379-85.

[9] Hinojosa-Moya J, Xoconostle-Cazares B, Piedra-Ibarra E, MendezTenorio A, Lucas WJ, Ruiz-Medrano R. Phylogenetic and structural analysis of translationally controlled tumor proteins. J Mol Evol 2008; 66(5): 472-83.

[10] Thaw P, Baxter NJ, Hounslow AM, Price C, Waltho JP, Craven CJ. Structure of TCTP reveals unexpected relationship with guanine nucleotide-free chaperones. Nat Struct Biol 2001; 8(8): 701-4.

[11] Haghighat NG, Ruben L. Purification of novel calcium binding proteins from Trypanosoma brucei: properties of 22-, 24- and 38kilodalton proteins. Mol Biochem Parasitol 1992; 51(1): 99-110.

[12] Graidist $\mathrm{P}$, Yazawa M, Tonganunt $\mathrm{M}$, et al. Fortilin binds $\mathrm{Ca} 2+$ and blocks Ca2+-dependent apoptosis in vivo. Biochem J 2007; 408(2): 181-91.

[13] Feng Y, Liu D, Yao H, Wang J. Solution structure and mapping of a very weak calcium-binding site of human translationally controlled tumor protein by NMR. Arch Biochem Biophys 2007; 467(1): 48-57.

[14] Gachet Y, Tournier S, Lee M, Lazaris-Karatzas A, Poulton T, Bommer UA. The growth-related, translationally controlled protein P23 has properties of a tubulin binding protein and associates transiently with microtubules during the cell cycle. J Cell Sci 1999; 112(Pt 8): 1257-71.

[15] Bazile F, Pascal A, Arnal I, Le Clainche C, Chesnel F, Kubiak JZ. Complex relationship between TCTP, microtubules and actin 
microfilaments regulates cell shape in normal and cancer cells. Carcinogenesis 2009; 30(4): 555-65.

[16] Tsarova K, Yarmola EG, Bubb MR. Identification of a cofilin-like actin-binding site on translationally controlled tumor protein (TCTP). FEBS Lett 2011; 584(23): 4756-60.

[17] Burgess A, Labbe JC, Vigneron S, et al. Chfr interacts and colocalizes with TCTP to the mitotic spindle. Oncogene 2008; 27(42): 5554-66.

[18] Kubiak JZ, Bazile F, Pascal A, et al. Temporal regulation of embryonic M-phases. Folia Histochem Cytobiol 2008;46(1):5-9.

[19] Yarm FR. Plk phosphorylation regulates the microtubulestabilizing protein TCTP. Mol Cell Biol 2002; 22(17): 6209-21.

[20] Johnson TM, Antrobus R, Johnson LN. Plk1 activation by Ste20like kinase (Slk) phosphorylation and polo-box phosphopeptide binding assayed with the substrate translationally controlled tumor protein (TCTP). Biochemistry 2008; 47(12): 3688-96.

[21] Cucchi U, Gianellini LM, De Ponti A, et al. Phosphorylation of TCTP as a marker for polo-like kinase-1 activity in vivo. Anticancer Res 2010; 30(12): 4973-85.

[22] Johansson H, Svensson F, Runnberg R, Simonsson T, Simonsson S. Phosphorylated nucleolin interacts with translationally controlled tumor protein during mitosis and with Oct4 during interphase in ES cells. PLoS One 2010; 5(10): e13678.

[23] Johansson H, Vizlin-Hodzic D, Simonsson T, Simonsson S. Translationally controlled tumor protein interacts with nucleophosmin during mitosis in ES cells. Cell Cycle 2010; 9(11): 2160-9.

[24] Chan TH, Chen L, Liu M, et al. Translationally controlled tumor protein induces mitotic defects and chromosome missegregation in hepatocellular carcinoma development. Hepatology 2012; 55(2): 491-505.

[25] Brioudes F, Thierry AM, Chambrier P, Mollereau B, Bendahmane M. Translationally controlled tumor protein is a conserved mitotic growth integrator in animals and plants. Proc Natl Acad Sci USA 2010; 107(37): 16384-9.

[26] Berkowitz O, Jost R, Pollmann S, Masle J. Characterization of TCTP, the translationally controlled tumor protein, from Arabidopsis thaliana. Plant Cell 2008; 20(12): 3430-47.

[27] Yan L, Fei K, Bridge D, Sarras MP, Jr. A cnidarian homologue of translationally controlled tumor protein (P23/TCTP). Dev Genes Evol 2000; 210(10): 507-11.

[28] Hsu Y-C, Chern JJ, Cai Y, Liu M, Choi KW. Drosophila TCTP is essential for growth and proliferation through regulation of $\mathrm{dRheb}$ GTPase. Nature 2007; 445(7129): 785-8.

[29] Chen SH, Wu PS, Chou $\mathrm{CH}$, et al. A knockout mouse approach reveals that TCTP functions as an essential factor for cell proliferation and survival in a tissue- or cell type-specific manner. Mol Biol Cell 2007; 18(7): 2525-32.

[30] Susini L, Besse S, Duflaut D, et al. TCTP protects from apoptotic cell death by antagonizing bax function. Cell Death Differ 2008; 15(8): 1211-20.

[31] Koide Y, Kiyota T, Tonganunt M, et al. Embryonic lethality of fortilin-null mutant mice by BMP-pathway overactivation. Biochim Biophys Acta 2009; 1790(5): 326-38

[32] Koziol MJ, Garrett N, Gurdon JB. Tpt1 activates transcription of oct4 and nanog in transplanted somatic nuclei. Curr Biol 2007; 17(9): 801-7.

[33] Tani T, Shimada H, Kato Y, Tsunoda Y. Bovine oocytes with the potential to reprogram somatic cell nuclei have a unique $23-\mathrm{kDa}$ protein, phosphorylated transcriptionally controlled tumor protein (TCTP). Cloning Stem Cells 2007; 9(2): 267-80.

[34] Guillaume E, Pineau C, Evrard B, et al. Cellular distribution of translationally controlled tumor protein in rat and human testes. Proteomics 2001; 1(7): 880-9.

[35] Vitale AM, Calvert MEK, Mallavarapu M, et al. Proteomic profiling of murine oocyte maturation. Mol Reprod Dev 2007; 74(5): 608-16

[36] Meyvis Y, Houthoofd W, Visser A, et al. Analysis of the translationally controlled tumour protein in the nematodes Ostertagia ostertagi and Caenorhabditis elegans suggests a pivotal role in egg production. Int J Parasitol 2009; 39(11): 1205-13.

[37] Li S, Chen X, Ding Y, Liu X, Wang Y, He J. Expression of translationally controlled tumor protein (TCTP) in the uterus of mice of early pregnancy and its possible significance during embryo implantation. Hum Reprod 2011; 26(11): 2972-80.

[38] Arcuri F, Papa S, Meini A, et al. The translationally controlled tumor protein is a novel calcium binding protein of the human placenta and regulates calcium handling in trophoblast cells. Biol Reprod 2005; 73(4): 745-51.

[39] Jung J, Kim M, Kim M-J, et al. Translationally controlled tumor protein interacts with the third cytoplasmic domain of $\mathrm{Na}, \mathrm{K}$ ATPase alpha subunit and inhibits the pump activity in HeLa cells. J Biol Chem 2004; 279(48): 49868-75

[40] Kim MJ, Kwon JS, Suh SH, et al. Transgenic overexpression of translationally controlled tumor protein induces systemic hypertension via repression of $\mathrm{Na}+\mathrm{K}+-\mathrm{ATPase}$. J Mol Cell Cardiol 2008; 44(1): 151-9.

[41] Gnanasekar M, Dakshinamoorthy G, Ramaswamy K Translationally controlled tumor protein is a novel heat shock protein with chaperone-like activity. Biochem Biophys Res Commun 2009 21; 386(2): 333-7.

[42] Bommer UA, Heng C, Perrin A, et al. Roles of the translationally controlled tumour protein (TCTP) and the double-stranded RNAdependent protein kinase, PKR, in cellular stress responses. Oncogene 2010; 29(5): 763-73.

[43] Diraison F, Hayward K, Sanders KL, et al. Translationally controlled tumour protein (TCTP) is a novel glucose-regulated protein that is important for survival of pancreatic beta cells. Diabetologia 2011; 54(2): 368-79.

[44] Xu A, Bellamy AR, Taylor JA. Expression of translationally controlled tumour protein is regulated by calcium at both the transcriptional and post-transcriptional level. Biochem J 1999; 342 (Pt3): 683-9.

[45] Graidist P, Phongdara A, Fujise K. Antiapoptotic protein partners fortilin and MCL1 independently protect cells from 5-fluorouracilinduced cytotoxicity. J Biol Chem 2004; 279(39): 40868-75.

[46] Nagano-Ito M, Banba A, Ichikawa S. Functional cloning of genes that suppress oxidative stress-induced cell death: TCTP prevents hydrogen peroxide-induced cell death. FEBS Lett 2009; 583(8): 1363-7.

[47] Gnanasekar M, Ramaswamy K. Translationally controlled tumor protein of Brugia malayi functions as an antioxidant protein. Parasitol Res 2007; 101(6): 1533-40.

[48] Lucibello M, Gambacurta A, Zonfrillo M, et al. TCTP is a critical survival factor that protects cancer cells from oxidative stress induced cell death. Exp Cell Res 2011; 317(17): 2479-89.

[49] Wu PS, Yang CY, Yen JJ, et al. Critical roles of translationally controlled tumor protein in the homeostasis and TCR-mediated proliferation of peripheral T cells. J Immunol 2009; 183(4): 2373 81.

[50] Xiong Z, Yan Y, Song J, et al. Expression of TCTP antisense in $\mathrm{CD} 25$ (high) regulatory $\mathrm{T}$ cells aggravates cuff-injured vascular inflammation. Atherosclerosis 2009; 203(2): 401-8.

[51] Liu H, Peng HW, Cheng YS, Yuan HS, Yang-Yen HF. Stabilization and enhancement of the antiapoptotic activity of mcl-1 by TCTP. Mol Cell Biol 2005; 25(8): 3117-26.

[52] Zhang D, Li F, Weidner D, Mnjoyan ZH, Fujise K. Physical and functional interaction between myeloid cell leukemia 1 protein (MCL1) and fortilin. The potential role of MCL1 as a fortilin chaperone. J Biol Chem 2002; 277(40): 37430-8.

[53] Yang Y, Yang F, Xiong Z, et al. An N-terminal region of translationally controlled tumor protein is required for its antiapoptotic activity. Oncogene 2005; 24(30): 4778-88

[54] Rinnerthaler M, Jarolim S, Heeren G, et al. MMI1 (YKL056c, TMA19), the yeast orthologue of the translationally controlled tumor protein (TCTP) has apoptotic functions and interacts with both microtubules and mitochondria. Biochim Biophys Acta 2006; 1757(5-6): 631-8.

[55] Sirois I, Raymond MA, Brassard N, et al. Caspase-3-dependent export of TCTP: a novel pathway for antiapoptotic intercellular communication. Cell Death Differ 2011; 18(3): 549-62.

[56] Tuynder M, Susini L, Prieur S, et al. Biological models and genes of tumor reversion: cellular reprogramming through tpt1/TCTP and SIAH-1. Proc Natl Acad Sci USA 2002; 99(23): 14976-81.

[57] Rho SB, Lee JH, Park MS, et al. Anti-apoptotic protein TCTP controls the stability of the tumor suppressor p53. FEBS Lett 2011; 585(1): 29-35. 
[58] Chen Y, Fujita T, Zhang D, et al. Physical and functional antagonism between tumor suppressor protein p53 and fortilin, an anti-apoptotic protein. J Biol Chem 2011; 286: 32575-85.

[59] Amson R, Pece S, Lespagnol A, et al. Reciprocal repression between P53 and TCTP. Nat Med 2011; 18(1): 91-9.

[60] Bommer UA, Borovjagin AV, Greagg MA, et al. The mRNA of the translationally controlled tumor protein P23/TCTP is a highly structured RNA, which activates the dsRNA-dependent protein kinase PKR. RNA 2002; 8(4): 478-96.

[61] Kim M, Jung J, Lee K. Roles of ERK, PI3 kinase, and PLC-gamma pathways induced by overexpression of translationally controlled tumor protein in HeLa cells. Arch Biochem Biophys 2009; 485(1): 82-7.

[62] Jung J, Kim HY, Kim M, Sohn K, Lee K. Translationally controlled tumor protein induces human breast epithelial cell transformation through the activation of Src. Oncogene 2011; 30(19):2264-74.

[63] Wang D, Gao L. Proteomic analysis of neural differentiation of mouse embryonic stem cells. Proteomics 2005; 5(17): 4414-26.

[64] Zhu WL, Cheng HX, Han N, et al. Messenger RNA expression of translationally controlled tumor protein (TCTP) in liver regeneration and cancer. Anticancer Res 2008; 28(3A): 1575-80.

[65] Proud CG. mTORC1 signalling and mRNA translation. Biochem Soc Trans 2009; 37(Pt1): 227-31.

[66] Wang X, Fonseca BD, Tang H, et al. Re-evaluating the roles of proposed modulators of mammalian target of rapamycin complex 1 (mTORC1) signaling. J Biol Chem 2008; 283(45): 30482-92.

[67] Rehmann $\mathrm{H}$, Bruning $\mathrm{M}$, Berghaus $\mathrm{C}$, et al. Biochemical characterisation of TCTP questions its function as a guanine nucleotide exchange factor for Rheb. FEBS Lett 2008; 582(20): 3005-10.

[68] Dong X, Yang B, Li Y, Zhong C, Ding J. Molecular basis of the acceleration of the GDP-GTP exchange of human ras homolog enriched in brain by human translationally controlled tumor protein. J Biol Chem 2009; 284(35): 23754-64.

[69] Yubero N, Esteso G, Cardona H, Morera L, Garrido JJ, Barbancho M. Molecular cloning, expression analysis and chromosome localization of the Tpt1 gene coding for the pig translationally controlled tumor protein (TCTP). Mol Biol Rep 2009; 36(7): 195765.

[70] Cans C, Passer BJ, Shalak V, et al. Translationally controlled tumor protein acts as a guanine nucleotide dissociation inhibitor on the translation elongation factor eEF1A. Proc Natl Acad Sci USA 2003; 100(24): 13892-7.

[71] Langdon JM, Vonakis BM, MacDonald SM. Identification of the interaction between the human recombinant histamine releasing factor/translationally controlled tumor protein and elongation factor-1 delta (also known as eElongation factor-1B beta). Biochim Biophys Acta 2004; 1688(3): 232-6.

[72] Leclercq TM, Moretti PA, Pitson SM. Guanine nucleotides regulate sphingosine kinase 1 activation by eukaryotic elongation factor $1 \mathrm{~A}$ and provide a mechanism for eEF1A-associated oncogenesis. Oncogene $2011 ; 30(3)$ : 372-8

[73] Thiele H, Berger M, Skalweit A, Thiele BJ. Expression of the gene and processed pseudogenes encoding the human and rabbit translationally controlled tumour protein (TCTP). Eur J Biochem 2000; 267(17): 5473-81.

[74] Sanchez JC, Schaller D, Ravier F, et al. Translationally controlled tumor protein: a protein identified in several nontumoral cells including erythrocytes. Electrophoresis 1997; 18(1): 150-5.

[75] Kim JE, Koo KH, Kim YH, Sohn J, Park YG. Identification of potential lung cancer biomarkers using an in vitro carcinogenesis model. Exp Mol Med 2008; 40(6): 709-20.

[76] Telerman A, Amson R. The molecular programme of tumour reversion: the steps beyond malignant transformation. Nat Rev Cancer 2009; 9(3): 206-16.

[77] Tuynder M, Fiucci G, Prieur S, et al. Translationally controlled tumor protein is a target of tumor reversion. Proc Natl Acad Sci USA 2004; 101(43): 15364-9.

[78] Ge F, Zhang L, Tao SC, et al. Quantitative proteomic analysis of tumor reversion in multiple myeloma cells. J Proteome Res 2011; 10(2): 845-55.

[79] Gnanasekar M, Thirugnanam S, Zheng G, Chen A, Ramaswamy K. Gene silencing of translationally controlled tumor protein (TCTP) by siRNA inhibits cell growth and induces apoptosis of human prostate cancer cells. Int J Oncol 2009; 34(5): 1241-6.

[80] Lee JH, Rho SB, Park SY, Chun T. Interaction between fortilin and transforming growth factor-beta stimulated clone-22 (TSC-22) prevents apoptosis via the destabilization of TSC-22. FEBS Lett 2008; 582(8): 1210-8.

[81] Ma Q, Geng Y, Xu W, et al. The role of translationally controlled tumor protein in tumor growth and metastasis of colon adenocarcinoma cells. J Proteome Res 2010; 9(1): 40-9.

[82] Yao Y, Jia XY, Tian HY, et al. Comparative proteomic analysis of colon cancer cells in response to oxaliplatin treatment. Biochim Biophys Acta 2009; 1794(10): 1433-40.

[83] Bhisutthibhan J, Pan XQ, Hossler PA, et al. The Plasmodium falciparum translationally controlled tumor protein homolog and its reaction with the antimalarial drug artemisinin. J Biol Chem 1998; 273(26): 16192-8.

[84] Chae J, Choi I, Kim C. Homology modeling and molecular docking study of translationally controlled tumor protein and artemisinin. Arch Pharmacol Res 2006; 29(1): 50-8.

[85] Efferth T. Mechanistic perspectives for 1,2,4-trioxanes in anticancer therapy. Drug Resist Updat 2005; 8(1-2): 85-97.

[86] Fujita T, Felix K, Pinkaew D, Hutadilok-Towatana N, Liu Z, Fujise $\mathrm{K}$. Human fortilin is a molecular target of dihydroartemisinin. FEBS Lett 2008; 582(7): 1055-60.

[87] Vercoutter-Edouart AS, Czeszak X, Crepin M, et al. Proteomic detection of changes in protein synthesis induced by fibroblast growth factor-2 in MCF-7 human breast cancer cells. Exp Cell Res 2001; 262(1): 59-68.

[88] Schmidt I, Fahling M, Nafz B, Skalweit A, Thiele BJ. Induction of translationally controlled tumor protein (TCTP) by transcriptional and post-transcriptional mechanisms. FEBS J 2007; 274(20): 541624.

[89] Baudet C, Perret E, Delpech B, et al. Differentially expressed genes in C6.9 glioma cells during vitamin D-induced cell death program. Cell Death Differ 1998; 5(1): 116-25.

[90] Rid R, Onder K, Trost A, et al. H2O2-dependent translocation of TCTP into the nucleus enables its interaction with VDR in human keratinocytes: TCTP as a further module in calcitriol signalling. J Steroid Biochem Mol Biol 2010; 118(1-2): 29-40.

[91] Rupec RA, Poujol D, Kaltschmidt C, Messer G. Isolation of a hypoxia-induced cDNA with homology to the mammalian growthrelated protein p23. Oncol Res 1998; 10(2): 69-74.

[92] Sturzenbaum SR, Kille P, Morgan AJ. Identification of heavy metal induced changes in the expression patterns of the translationally controlled tumour protein (TCTP) in the earthworm Lumbricus rubellus1. Biochim Biophys Acta 1998; 1398(3): 294-304.

[93] Ermolayev V, Weschke W, Manteuffel R. Comparison of Alinduced gene expression in sensitive and tolerant soybean cultivars. J Exp Bot 2003; 54(393): 2745-56.

[94] Mak CH, Poon MW, Lun HM, Kwok PY, Ko RC. Heat-inducible translationally controlled tumor protein of Trichinella pseudospiralis: cloning and regulation of gene expression. Parasitol Res 2007; 100(5): 1105-11.

[95] Mak CH, Su KW, Ko RC. Identification of some heat-induced genes of Trichinella spiralis. Parasitology 2001; 123(Pt 3): 293300 .

[96] Cao B, Lu Y, Chen G, Lei J. Functional characterization of the translationally controlled tumor protein (TCTP) gene associated with growth and defense response in cabbage. Plant Cell Tissue Organ Cult 2010; 103: 217-26.

[97] Yan Y, Weaver VM, Blair IA. Analysis of protein expression during oxidative stress in breast epithelial cells using a stable isotope labeled proteome internal standard. J Proteome Res 2005; 4(6): 2007-14.

[98] Oikawa K, Ohbayashi T, Mimura J, et al. Dioxin stimulates synthesis and secretion of IgE-dependent histamine-releasing factor. Biochem Biophys Res Commun 2002; 290(3): 984-7.

[99] Zheng S, Song Y, Qiu X, Sun T, Ackland ML, Zhang W. Annetocin and TCTP expressions in the earthworm Eisenia fetida exposed to PAHs in artificial soil. Ecotoxicol Environ Saf 2008; 71(2): 566-73.

[100] Thiele H, Berger M, Lenzner C, Kuhn H, Thiele BJ. Structure of the promoter and complete sequence of the gene coding for the 
rabbit translationally controlled tumor protein (TCTP) P23. Eur J Biochem 1998; 257(1): 62-8.

[101] Fiucci G, Lespagnol A, Stumptner-Cuvelette P, et al. Genomic organization and expression of mouse Tpt1 gene. Genomics 2003; 81(6): 570-8.

[102] Bonnet C, Perret E, Dumont X, Picard A, Caput D, Lenaers G. Identification and transcription control of fission yeast genes repressed by an ammonium starvation growth arrest. Yeast 2000; 16(1): 23-33.

[103] Andree H, Thiele H, Fahling M, Schmidt I, Thiele BJ. Expression of the human TPT1 gene coding for translationally controlled tumor protein (TCTP) is regulated by CREB transcription factors. Gene 2006; 380(2): 95-103.

[104] Clemens MJ, Bommer UA. Translational control: the cancer connection. Int J Biochem Cell Biol 1999; 31(1): 1-23.

[105] Bommer UA, Lazaris-Karatzas A, De Benedetti A, et al. Translational regulation of the mammalian growth-related protein P23: involvement of eIF-4E. Cell Mol Biol Res 1994; 40(7-8): 63341.

[106] Robert F, Pelletier J. Translation initiation: a critical signalling node in cancer. Expert Opin Ther Targets 2009; 13(11): 1279-93.
[107] Fritsch RM, Schneider G, Saur D, Scheibel M, Schmid RM. Translational repression of MCL-1 couples stress-induced eIF2 alpha phosphorylation to mitochondrial apoptosis initiation. J Biol Chem 2007; 282(31): 22551-62.

[108] Efferth T. Molecular pharmacology and pharmacogenomics of artemisinin and its derivatives in cancer cells. Curr Drug Targets 2006; 7(4): 407-21.

[109] Chung S, Kim M, Choi W, Chung J, Lee K. Expression of translationally controlled tumor protein mRNA in human colon cancer. Cancer Lett 2000; 156(2): 185-90.

[110] Kuramitsu Y, Nakamura K. Proteomic analysis of cancer tissues: shedding light on carcinogenesis and possible biomarkers. Proteomics 2006; 6(20): 5650-61.

[111] Deng SS, Xing TY, Zhou HY, et al. Comparative proteome analysis of breast cancer and adjacent normal breast tissues in human. Genomics Proteomics Bioinformatics 2006; 4(3): 165-72.

[112] Slaby O, Sobkova K, Svoboda M, et al. Significant overexpression of Hsp110 gene during colorectal cancer progression. Oncol Rep 2009; 21(5): 1235-41.

Received: January 25, 2012

Revised: February 10, 2012

Accepted: February 17, 2012

(C) Ulrich-Axel Bommer; Licensee Bentham Open.

This is an open access article licensed under the terms of the Creative Commons Attribution Non-Commercial License (http://creativecommons.org/licenses/by-nc/3.0/) which permits unrestricted, non-commercial use, distribution and reproduction in any medium, provided the work is properly cited. 